\title{
Uma Experiência com a Utilização de Sistemas ERP para Auxiliar a Aquisição de Conhecimento sobre Processos de Negócio em um Curso de Bacharelado em Administração Alternative Title: An Experiment with the ERP Systems usage to assist the Knowledge Acquisition of Business Processes in Bachelors on Business Administration Course
}

\author{
Márcio Pires de Oliveira \\ Faculdade de Economia, Administração e \\ Contabilidade de Ribeirão Preto - USP \\ Avenida Bandeirantes, 3900 \\ Monte Alegre, 14040-905 - Ribeirão Preto, SP-Brasil \\ +55 (16) 3636-3338 \\ mpoliveira@fearp.usp.br
}

\author{
Ildeberto Aparecido Rodello \\ Faculdade de Economia, Administração e \\ Contabilidade de Ribeirão Preto - USP \\ Avenida Bandeirantes, 3900
}

Monte Alegre, 14040-905 - Ribeirão Preto, SP-Brasil

+55 (16) 3315-0514

rodello@fearp.usp.br

\begin{abstract}
RESUMO
$\mathrm{O}$ uso de sistemas ERP no processo de ensino/aprendizado para estudantes do curso de Administração é uma ferramenta importante na aquisição de conhecimento relativo aos processos das empresas e à visão integrada destes processos. Diversos autores defendem que o ensino de sistemas ERP pode ser favorecido com a aplicação da metodologia hands-on, na qual o estudante constrói seu conhecimento por meio de ferramentas fornecidas pelo educador. O objetivo do presente estudo é relatar uma experiência realizada com uma turma de 68 alunos com o uso de sistemas ERP na abordagem hands-on como ferramenta auxiliar no processo de ensino/aprendizagem em um curso de Administração. A avaliação da utilização dessa abordagem se deu por meio de uma metodologia que visa comparar a situação do conhecimento dos alunos relativo a um processo geral de vendas antes e depois de um exercício hands-on. Foi possível observar uma melhora do conhecimento sobre o processo estudado, evidenciada num maior entendimento de quais são os atores envolvidos e maior coerência e clareza nas relações entre estes atores e das atividades por eles realizadas.
\end{abstract}

\section{Palavras-chave}

Sistemas ERP, aprendizado hands-on.

\begin{abstract}
The ERP systems usage for the teaching/learning process to business administration students is an important tool in the acquisition of knowledge about business process integration view. Several authors have stated that the learning of ERP systems could be favored by the application of the hands on methodology, on which the student build your knowledge through tools provided by educator. The objective of this paper is describe an experience with
\end{abstract}

Permission to make digital or hard copies of all or part of this work for personal or classroom use is granted without fee provided that copies are not made or distributed for profit or commercial advantage and that copies bear this notice and the full citation on the first page. To copy otherwise, or republish, to post on servers or to redistribute to lists, requires prior specific permission and/or a fee.

SBSI 2015, May 26-29, 2015, Goiânia, Goiás, Brazil.

Copyright SBC 2015. a 68 students about the ERP system usage according to hands-on approach as a tool to facilitate the teaching/learning process in bachelors on business administration course. The analysis of this approach was made through a methodology based on comparison of a student's knowledge related to a general sales process before and after of hands-on exercise application. It was possible to observe an improvement of knowledge about the sales process, highlighted through the comprehension of the involved actors as well as the relationship between them and the executed tasks.

\section{Categories and Subject Descriptors}

K.3 [Computers and Education]: Computer and Information Science Education - Information systems education

\section{General Terms \\ Experimentation}

Keywords

ERP systems, hands-on learning.

\section{INTRODUÇÃO}

As empresas estão inseridas em um ambiente cada vez mais competitivo e em constante mudança. O surgimento de novas tecnologias tem um papel fundamental no direcionamento dessas mudanças, em geral deslocando a vantagem competitiva para aqueles que estejam na vanguarda do desenvolvimento.

Na década de 1970, algumas empresas começaram a utilizar a tecnologia computacional para melhor integrar e controlar suas operações [13]. Os computadores foram tornando-se cada vez mais baratos e seu uso cada vez mais popular.

Com o desenvolvimento da tecnologia, surgiram pacotes de soluções de software mais robustos e que buscavam integrar toda a gama de processos e funções de um negócio, objetivando oferecer uma visão holística a partir de uma única arquitetura de Tecnologia de Informação (TI) e de informação. Esses pacotes de sistemas receberam o nome de Enterprise Resource Planning systems (ERPs), embora haja alguma controvérsia em relação à aplicabilidade do termo [7].

Na década de 1990, os sistemas ERP foram o foco da área de TI ao redor do mundo, quando foi observado um aumento expressivo na utilização desses sistemas [2]. Com o tempo, a complexidade dos 
processos desenvolvidos pelas empresas em meio a este ambiente cada vez mais competitivo fez com que os sistemas ERP deixassem de ser uma ferramenta criadora de vantagem competitiva para tornarem-se uma necessidade, um requisito para apenas estar a par dos outros competidores.

Com o desenvolvimento, funções facilitadas pelos ERP, como coordenação funcional, comunicação efetiva e integração de processos tornaram-se essenciais para as operações de negócio [13]. Neste contexto de crescente competitividade, uma melhor compreensão e transparência nos processos operacionais de negócio são essenciais para praticamente qualquer empresa [1].

Observa-se, portanto, que o ensino de sistemas ERP em cursos de Administração pode ter importância para favorecer a compreensão do funcionamento dos diferentes processos das empresas, bem como suporte para a integração desses processos.

A inclusão do tema sistemas ERP em cursos superiores na área de negócios justifica-se por diversos motivos, tais como: o aprendizado de ERP é uma habilidade importante de TI; as organizações precisam de pessoas sagazes no uso de ERP em suas atividades diárias; recentes fracassos documentados de projetos de ERP sugerem que o conhecimento e as habilidades relacionadas aos ERP ainda são importantes e necessários. Além disso, o conhecimento sobre a utilização de sistemas ERP é requisito para algumas vagas de emprego [1].

Em relação à estratégia de ensino voltada para a informática, é oferecida uma posição favorável à utilização de uma abordagem hands-on. De Acordo com [5], a utilização desta metodologia não trata de oferecer ao estudante um objeto a ser construído simplesmente para que seja construído. Esta construção, de um artefato qualquer, deve ser escolhida pelo estudante, de forma que a construção será pessoalmente significativa, possibilitando um envolvimento afetivo entre o aprendiz e aquilo que se pretende produzir. Além disso, deve-se buscar produzir algo que seja tangível, que cuja construção seja realizável e compreensível pelo estudante.

Em um contraponto discutido por [13], não foram encontradas evidências de que uma intervenção de aprendizado hands-on produz resultados estatisticamente significativos no que diz respeito ao conhecimento detalhado dos estudantes relativo aos processos de negócios das empresas quando esta avaliação foi feita por meio de uma metodologia quantitativa e objetiva [13]. Por outro lado, há evidências de que a auto avaliação dos alunos relativa ao conhecimento próprio sobre os processos de negócio das empresas e a interconexão dos mesmos é beneficiada pela experiência de utilização de sistemas ERP [10, 13].

A utilização da abordagem hands-on como ferramenta auxiliar no processo de ensino de sistemas ERP passa pela dificuldade do custo elevado das soluções comerciais disponíveis. Dessa forma, optou-se por utilizar um sistema ERP open source, uma vez que a utilização de um software comercial licenciado necessita de parcerias comerciais que muitas vezes não são financeiramente viáveis para as Instituições de Ensino. Além disso, não há grande diferença no aprendizado relativo aos sistemas ERP e aos processos de negócios mais gerais ao utilizar diferentes sistemas, uma vez que tais processos estão implementados de acordo com as melhores práticas. Sendo assim, a utilização de uma ferramenta gratuita e amplamente disponível torna mais fácil a aplicação da abordagem hands-on para o ensino de sistemas ERP [1].

Dentro desse contexto, este estudo tem por objetivo relatar uma experiência com a utilização de sistemas ERP por estudantes de um curso de Administração em uma Instituição de Ensino Superior Pública, focalizando a compreensão sistêmica de integração dos processos da empresa e o detalhamento das etapas e pessoas envolvidas nestes processos. A estratégia de ensino adotada foi a hands-on.

Para melhor apresentar o objetivo mencionado anteriormente, este artigo é composto, além da introdução, de quatro seções, a saber: 2. Trabalhos Relacionados, 3. Metodologia e 4. Resultados, além da Bibliografia. Na seção de Trabalhos Relacionados estão descritas algumas pesquisas estudadas que se relacionam ao tema da pesquisa - ensino de sistemas ERP e a utilização da abordagem hands-on, além de uma breve apresentação do sistema OpenERP. $\mathrm{Na}$ Metodologia são caracterizados o tipo da pesquisa, o modelo para coleta de dados e o método de análise. Por fim, são apresentados os resultados obtidos e as discussões pertinentes ao presente estudo.

\section{TRABALHOS RELACIONADOS}

Esta seção está dividida com base nos principais temas que dão embasamento ao trabalho. Inicialmente, será feita uma revisão dos estudos realizados a respeito do tema de ensino de sistemas ERP. Em seguida, será trabalhado o conceito de aprendizado hands-on. Por fim, será feita uma breve apresentação do sistema OpenERP.

\subsection{A Importância do Ensino de Sistemas ERP}

Ao longo da última década, foram realizadas diversas tentativas de integração do currículo universitário utilizando sistemas ERP. Essas experiências levaram ao surgimento de pesquisas com foco nas técnicas pedagógicas, assim como no treinamento e aprendizado efetivos desse tipo de sistema [13].

As características dos sistemas ERP podem aflorar uma pedagogia mais efetiva na Educação Superior, principalmente para cursos da área de negócios, proporcionando uma compreensão mais aprofundada das operações e uma visão mais clara dos aspectos interligados da atividade de negócio [6].

Tal visão foi corroborada por um estudo que sugeriu que estudantes que passaram por um treinamento sobre os módulos de Recursos Humanos de um sistema ERP desenvolveram um melhor conhecimento sobre as práticas na área em comparação aos alunos que não passaram por este treinamento [16].

A utilização de sistemas ERP no Ensino Superior também pode exercer uma influência positiva no aprendizado de processos de negócio quando este aprendizado é medido pela auto avaliação dos estudantes [10]. Além disso, foram documentadas melhorias significativas na compreensão dos estudantes relativa à integração dos processos de negócios ao incluir a configuração de um software ERP no currículo [3].

Também foi levada em consideração uma investigação conduzida dos artigos pedagógicos sobre ERP produzidos no período de 2000 - 2010 e publicados no JISE (Journal of Information Systems Education) [1]. O fato de se tratar de uma revista especializada no ensino de sistemas de informação proporcionou uma boa amplitude de artigos mesmo limitando a busca a apenas uma revista. Foram identificados dois temas centrais para os artigos estudados: o primeiro grupo era de artigos focalizados em fornecer orientações sobre como integrar o item aos currículos universitários e sobre o ensino de ERP. O segundo grupo, por sua vez, era de artigos voltados para a importância do ensino dos conceitos dos sistemas ERP [1]

Em um outro experimento, no qual os estudantes participaram de uma competição de simulação de empresas, foram encontradas outras indicações de que a utilização de sistemas ERP em situações de simulação podem favorecer a compreensão dos estudantes sobre os processos das empresas e sua integração. Utilizando um sistema ERP real, o mySAP, os estudantes deveriam interpretar os relatórios e utilizar os módulos de inteligência de negócios para tomar decisões de negócios. Após a participação neste 
experimento, gradualmente os estudantes perceberam que reorganizar de acordo com um processo de negócio as tarefas que deveriam realizar permitiria a eles tirar proveito do sistema integrado. Além disso, os alunos puderam, ao final do experimento, compreender como o sistema integrado poderia auxiliar na obtenção de resultados [8].

Um conhecimento importante para os graduandos de cursos relacionados à área de negócios é a compreensão verdadeira do que é um processo de negócio - a unidade básica das operações envolvidas nos fluxos de trabalho das empresas, e em todas as operações de negócios [4]. Neste contexto, destaca-se a importância de aprender os conceitos fundamentais de processos de negócios para todos os graduandos de cursos da área de negócios [13].

Em um experimento realizado com o objetivo de medir o ganho de conhecimento relativo aos processos de venda e de compra de empresas utilizando o aprendizado hands-on em um sistema ERP, os resultados indicaram que, apesar de não ter sido encontrada significância estatística para este aumento de conhecimento, a auto avaliação dos alunos em relação ao seu conhecimento dos processos melhorou significativamente [13].

Em relação ao distribuidor do software utilizado no ensino de sistemas ERP, a maioria dos artigos relacionados ao ensino de sistemas ERP publicados anteriormente utilizava soluções comerciais, principalmente o SAP. Esse viés pode dificultar a implementação de uma metodologia hands-on, pois necessita de parcerias comerciais que muitas vezes não são financeiramente viáveis para as Instituições de Ensino. Além disso, o aprendizado relativo aos processos de negócios e à integração destes não é diferente quando da utilização de diferentes sistemas. Os sistemas ERP são desenvolvidos e implementados com base nas melhores práticas do mercado, de forma que há grande padronização. Sendo assim, o aprendizado em um sistema específico é válido para qualquer outro que seja igualmente baseado nas melhores práticas[1].

Dessa forma, o uso de um software gratuito e open source para o ensino de sistemas ERP em cursos de graduação é uma alternativa que desonera a utilização de uma abordagem hands-on.

\subsection{A Metodologia Hands-on}

A metodologia hands-on de aprendizado deriva dos princípios da teoria denominada construcionista, que vem sendo elaborada desde o final da década de 1960 , com a criação da linguagem LOGO por Seymour Papert. Esta teoria deriva de uma tradição da psicologia construtivista de Jean Piaget, dentre outros teóricos, uma vez que atribui aos aprendizes o papel de construtores do próprio conhecimento [5].

A metodologia de aprendizagem hands-on visa criar a ponte necessária para a ligação do conhecimento teórico e o ambiente de aprendizado à solução prática de problemas reais, embora os limites exatos da teoria sejam deliberadamente fluidos na literatura [5]. O educador desempenha um papel chave na medida em que fornece a orientação e os instrumentos necessários ao aluno para a solução destes problemas e, consequentemente, para a construção de conhecimento. Neste contexto, destaca-se a característica da metodologia hands-on de oferecer ao estudante as ferramentas necessárias para que o conhecimento seja construído por ele mesmo.

$\mathrm{O}$ uso de uma metodologia hands-on pode ser benéfica para o ensino de sistemas ERP e para a compreensão dos processos de negócios. A utilização de uma abordagem de aprendizado handson experimental envolve ativamente os estudantes no processo de aprendizagem. Ao desempenhar atividades em um ERP real, os estudantes desenvolvem suas habilidades específicas àquele ERP, mas também compreendem melhor as funcionalidades de sistemas
ERP como um todo e adquirem uma visão da integração dos processos de negócios [13].

Em um estudo voltado para a aproximação entre academia e indústria, foi utilizada uma abordagem prática para aprimorar o ensino de Engenharia de Software em um curso superior de Tecnologia em Análise e Desenvolvimento de Sistemas. Ao substituir os projetos fictícios realizados na disciplina por projetos reais de empresas, as autoras destacam, em relação ao entendimento dos alunos sobre o processo de desenvolvimento de software, que as atividades do processo e o relacionamento entre elas tornaram-se mais claros e adequados, além das entradas, saídas e orientações em geral [15]

Desse modo, é possível perceber que o uso de uma metodologia como a hands-on possibilita melhor integração entre teoria e prática, tentando preencher a lacuna existente entre a teoria ensinada em sala de aula e as necessidades do mercado de trabalho.

\subsection{O Sistema OpenERP}

OpenERP é um pacote de software organizado em módulos oferecido gratuitamente no formato open source. O modelo de negócios adotado pela empresa que oferece o produto gera receita por meio da prestação de serviços ao invés da cobrança de licenças de uso [1].

Sob essa perspectiva, desenvolveu-se uma comunidade de desenvolvedores que constantemente melhoram o sistema e oferecem diferentes funcionalidades e customizações. A própria empresa que oferece o sistema também oferece gratuitamente guias e manuais para facilitar a inserção de novos usuários e encorajar o crescimento da comunidade de desenvolvedores.

Dentre as vantagens para uma empresa em utilizar o sistema OpenERP, destacam-se: não há cobrança de licenças de uso; a forma de licença do sistema permite que ele seja customizado pela empresa de forma livre; esse modelo de licença também encoraja a formação de uma comunidade de desenvolvedores, com o compartilhamento de informações e de soluções [9].

Atualmente, o sistema oferece diversos módulos categorizados em contabilidade, recursos humanos, localização, produção, projetos, compras, vendas, estoque e ferramentas extras [12].

\section{METODOLOGIA}

Esta seção divide-se em três partes. Primeiro, a pesquisa é caracterizada. Em seguida, é apresentada a maneira como foi realizada a coleta dos dados. Posteriormente, será discutido o método de análise que foi utilizado para a avaliação dos dados obtidos.

\subsection{Tipo de Estudo}

O presente estudo possui caráter exploratório, uma vez que consiste numa investigação inicial da utilização de um sistema ERP, utilizando a abordagem hands-on, no auxílio do processo de ensinagem de sistemas integrados de gestão para estudantes do curso de bacharelado em Administração.

\subsection{Procedimento para Coleta de Dados}

A fim de contemplar os objetivos deste estudo, foi escolhida como população alvo uma turma do $6^{\circ}$ semestre do curso de bacharelado em Administração da Faculdade de Economia, Administração e Contabilidade da USP de Ribeirão Preto (FEA-RP), cursando a disciplina RAD1608 - Sistemas Integrados de Informação ao momento do estudo. Este grupo totaliza 68 alunos matriculados no período diurno. $\mathrm{O}$ experimento realizado teve duração de 3 aulas, obedecendo o seguinte protocolo.

Inicialmente, os estudantes receberam uma orientação quanto às 
notações para elaboração de um diagrama de atividades segundo a Unified Modeling Language (UML). A escolha por esse diagrama se pautou no fato de que eles já haviam tido contato com a notação em outra disciplina cursada anteriormente. O diagrama de atividades oferece uma notação padronizada para modelagem de processos que permite a fácil visualização dos atores envolvidos no processo e das atividades relacionadas. A UML foi desenvolvida pelo Object Management Group (OMG) com o objetivo de unificar cada passo do desenvolvimento e integração, desde a modelagem de processos, passando pela arquitetura e modelagem de aplicações, até o desenvolvimento, lançamento, manutenção e evolução [11].

Os estudantes foram então divididos em grupos de quatro a seis integrantes, que permaneceram os mesmos durante todo o experimento. Cada grupo foi então orientado a modelar o processo geral de vendas de uma empresa utilizando as noções obtidas do diagrama de atividades.

No segundo dia do experimento, os estudantes tiveram o primeiro contato com o sistema OpenERP. O instrutor fez demonstrações de uso do sistema e em seguida os estudantes tiveram algum tempo para utilizarem o sistema sozinhos e familiarizarem-se com sua interface, sem a preocupação da realização de uma atividade específica. O objetivo foi permitir que os estudantes se familiarizassem com a interface do sistema.

No terceiro dia foi realizado o exercício hands-on proposto no experimento. Os estudantes, em seus respectivos grupos, desempenharam por conta própria todo o processo geral de vendas de uma empresa utilizando o sistema OpenERP.

O sistema já estava pré-configurado com alguns clientes e produtos cadastrados. Para a realização do exercício, foram criados três perfis diferentes no sistema, além do perfil do administrador do sistema, acessado apenas pelos pesquisadores para fins de acompanhamento. Os estudantes receberam acesso aos perfis de Vendedor, Financeiro e Suprimentos, cada um deles com autorizações de acesso diferentes. O exercício foi diferente para cada grupo, ora seria vendido um determinado produto, ora algum outro, mas o processo foi sempre o mesmo.

Em linhas gerais, o primeiro passo do processo que foi desempenhado pelos alunos era a criação de um novo cliente para o qual seria realizada a venda. Uma vez criado o cliente, os estudantes criaram um pedido de venda utilizando o perfil Vendedor, selecionando o cliente criado e o produto e quantidade a serem vendidos. Em seguida, o perfil Suprimentos realizou a checagem da disponibilidade do produto e confirmou o pedido. Uma vez confirmada o pedido, o perfil Financeiro emitiu a fatura, confirmou o pagamento e emitiu a nota fiscal. Após confirmar o pagamento, o perfil Suprimentos criou o pedido de entrega e confirmou a entrega. Por fim, o perfil Vendedor concluiu a venda. A Figura 1 mostra uma modelagem feita pelos pesquisadores ilustrando o processo descrito anteriormente

Após a realização do exercício, os grupos foram novamente convidados a modelar o processo geral de vendas de uma empresa, obedecendo a notação do diagrama de atividades da UML.

A primeira modelagem do processo geral de vendas feita pelos grupos serviu como a base comparativa para avaliar a evolução do conhecimento dos alunos evidenciada na segunda modelagem, feita após o desempenho do exercício hands-on no sistema OpenERP.

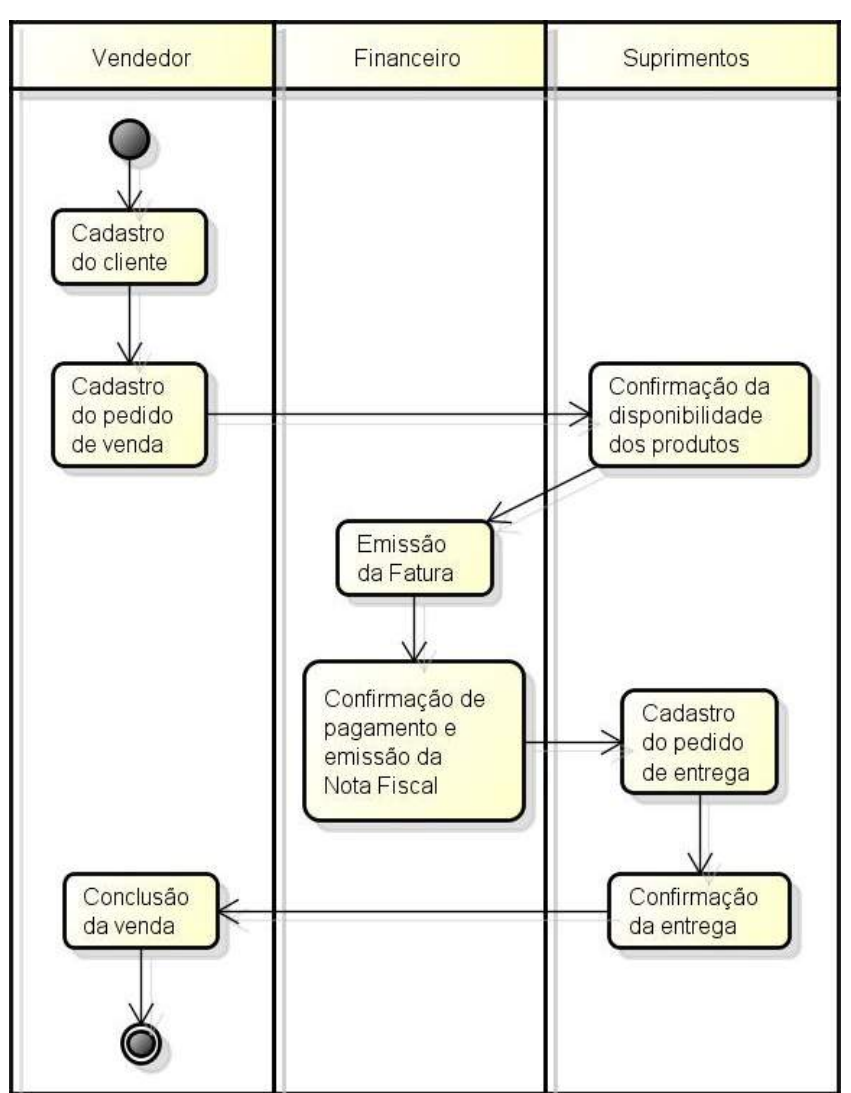

Figura 1 - Modelagem do processo geral de vendas utilizado no experimento

\subsection{Método de Análise}

Com a finalidade de avaliar a evolução do conhecimento relativo ao processo de vendas, as modelagens feitas pelos estudantes após o exercício hands-on foram comparadas às modelagens feitas antes do início do experimento.

Apesar de subjetiva, a análise foi pautada em parâmetros préconcebidos e considerou não só a estrutura montada como um todo, mas também questões mais específicas. A comparação entre as modelagens feitas pelos grupos antes e depois do exercício handson foi feita buscando melhoras no que diz respeito à coerência e à quantidade dos atores envolvidos e dos processos e atividades mostrados. Além disso, buscou-se também identificar melhorias na clareza e coerência nos relacionamentos lógicos estabelecidos entre os diferentes atores.

\section{RESULTADOS}

Foram compostos quinze grupos de quatro a seis integrantes, totalizando os 68 estudantes participantes do experimento. Do total de participantes, $51,5 \%$ são do sexo feminino. $38,2 \%$ dos participantes nunca tiveram um emprego formal, mas 39,7\% já havia feito estágio e 5,9\% já havia sido empregado informalmente. Somente 16,2\% dos estudantes já haviam sido empregados formalmente. Além disso, 63,2\% dos participantes responderam nunca ter utilizado um sistema ERP anteriormente.

Apesar de haver quinze grupos, foram considerados para o estudo, entretanto, apenas nove dos quinze grupos. Os diagramas de atividades feitos por quatro dos grupos foram descartados por problemas na compreensão do exercício - o modelo de diagrama de atividades utilizado não foi o UML ou o processo desenhado não se tratava de um processo geral de vendas, mas de um processo 
específico (um dos grupos desenhou o processo de venda de uma farmácia de manipulação, por exemplo). Dois dos grupos participantes não estiveram presentes em todas as etapas do experimento, portanto também tiveram de ser descartados.

De forma geral, foi possível observar certa melhora nas características pertinentes - a quantidade e coerência na escolha dos atores e processos/atividades envolvidos e o relacionamento estabelecido entre esses atores e processos/atividades.

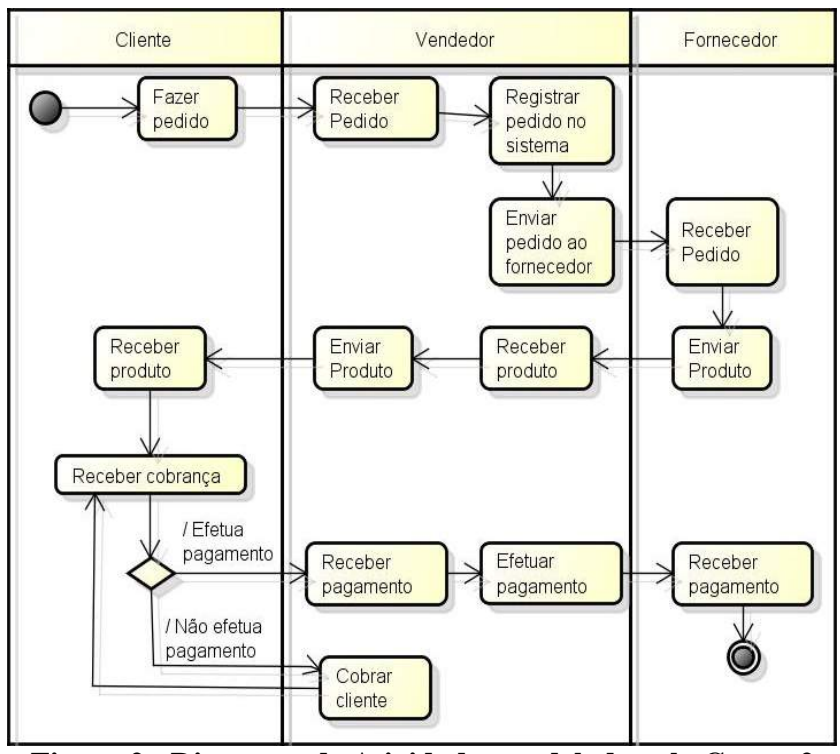

Figura 2 - Diagrama de Atividades modelado pelo Grupo 2 antes da utilização do sistema ERP.

Nos primeiros diagramas desenhados pelos grupos havia uma grande quantidade de atividades que eram irrelevantes ou não pertenciam propriamente a um processo geral de vendas. A ocorrência de atividades irrelevantes ou confusas reduziu bastante após os grupos desempenharem o processo de vendas usando o sistema ERP. A Figura 2 apresenta a modelagem de um grupo que incluiu em seu primeiro diagrama uma grande quantidade de atividades que foram removidas no segundo diagrama - atividades como recebimentos e envios redundantes, além de um ciclo de cobrança que inclui a possibilidade do não pagamento e emissão de nova cobrança ao cliente.

Na Figura 3 também é possível observar uma maior coerência na escolha dos atores envolvidos no processo: enquanto no primeiro diagrama (Figura 2), a empresa está representada apenas pelo "Vendedor", no segundo diagrama (Figura 3) aparecem os atores "Contador" e "Suprimentos". Além disso, foram incluídas atividades importantes, como a emissão da nota fiscal e a verificação da disponibilidade do produto, que não estão presentes no primeiro diagrama (Figura 2 ).

Aliás, é importante destacar que a inclusão da atividade de emissão da nota fiscal na modelagem do segundo diagrama foi observada em sete dos nove grupos considerados, sendo que apenas dois destes já incluíram a atividade desde a primeira modelagem. Houve alguma confusão em relação à posição desta atividade, mas foi possível observar que houve uma melhora em comparação à situação inicial.

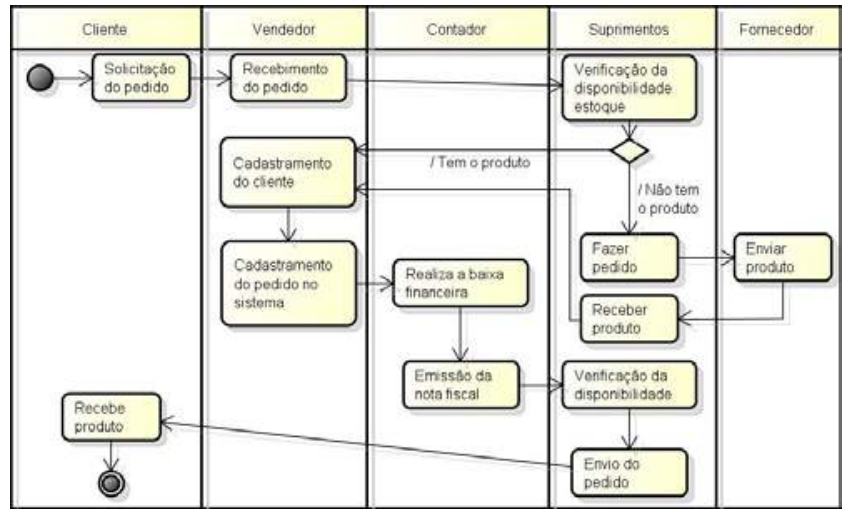

Figura 3 - Diagrama de Atividades modelado pelo Grupo 2 depois da utilização do sistema ERP.

Outra atividade importante que passou a ser incluída por alguns grupos foi a checagem de disponibilidade do produto. Seis grupos incluíram esta atividade no segundo diagrama, enquanto apenas dois destes grupos já incluíram a atividade desde o primeiro diagrama. A atividade de emitir a fatura do pedido, desempenhada pelos grupos durante o exercício, foi incluída no segundo diagrama por quatro dos grupos participantes.

Deve-se destacar, no entanto, que permaneceu certa confusão no subprocesso de faturamento do pedido para alguns dos grupos. A Figura 4 ilustra essa situação, em que o grupo inclui a atividade de emissão da nota fiscal, no entanto considera a nota fiscal e a fatura como o mesmo documento.

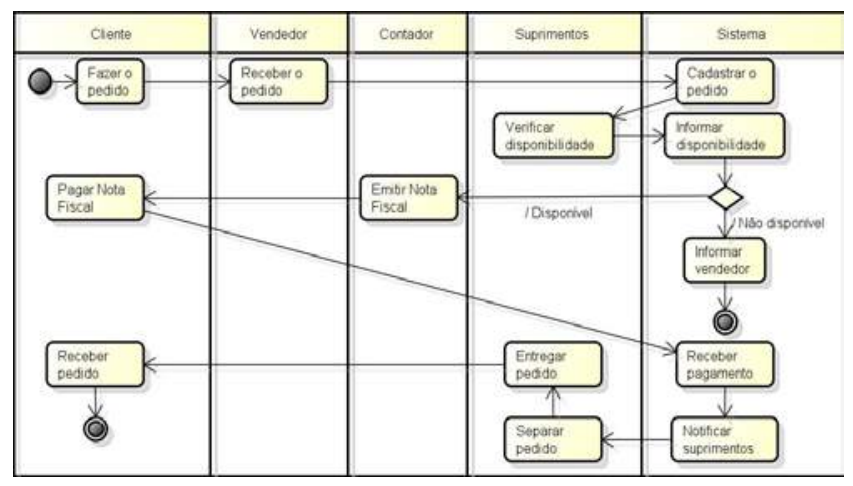

Figura 4 - Diagrama de Atividades modelado pelo Grupo 8 depois da utilização do sistema ERP.

Em relação à coerência na escolha das atividades/processos pelos grupos, foi possível observar uma maior clareza no diagrama elaborado ao final do experimento. As Figuras 5 e 6 mostram a evolução de um grupo nesse sentido - foram excluídas algumas atividades irrelevantes, como o cliente entrar na loja e o vendedor abordar o cliente (Figura 5), e foram incluídas atividades importantes como a emissão da nota fiscal e a checagem da disponibilidade do produto (Figura 6). 


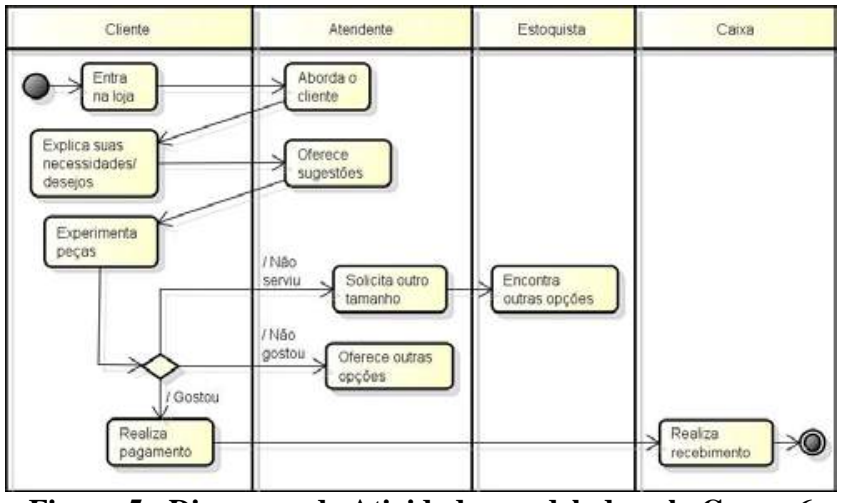

Figura 5 - Diagrama de Atividades modelado pelo Grupo 6 antes da utilização do sistema ERP.

Em relação aos atores envolvidos no processo, verificou-se uma grande variação nas escolhas feitas pelos grupos na primeira modelagem. Constavam diversos atores entre os grupos: "Cliente", "Financeiro/Contador", "Vendedor/Vendas", "Caixa", "Estoquista", "Fornecedor/Produção", "Entrega/Logística", "Expedição" e "Sistema". Já nos diagramas elaborados pelos grupos ao final do experimento, houve uma grande padronização constavam principalmente o "Cliente", "Vendedor/Vendas", "Contador/Financeiro" e "Suprimentos", talvez por influência da nomenclatura utilizada pelo sistema ERP. Apareceram também em um dos grupos o ator "Sistema" e em outro o "Fornecedor". Esta padronização pode ser entendida como influência direta do exercício, uma vez que os atores escolhidos no segundo momento eram aqueles claramente envolvidos no processo de vendas desempenhado pelos grupos

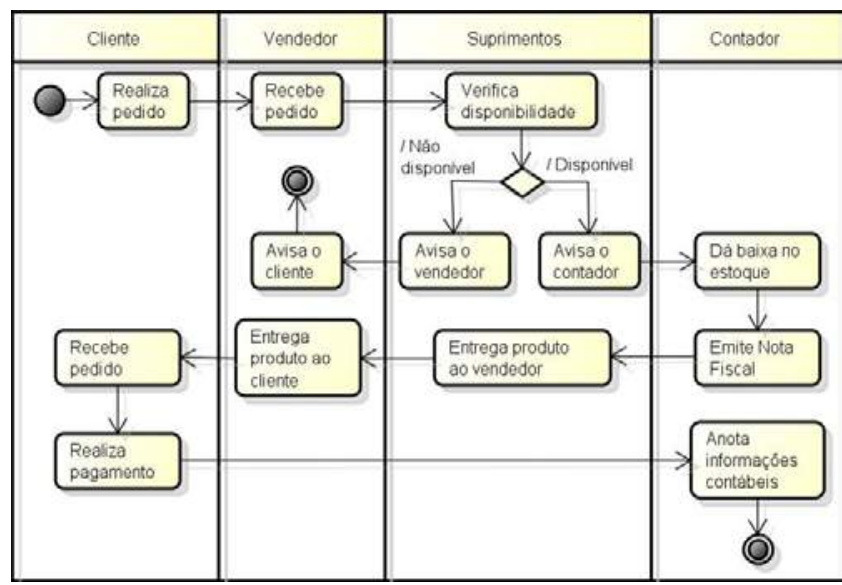

Figura 6 - Diagrama de Atividades modelado pelo Grupo 6 depois da utilização do sistema ERP.

A escolha dos atores envolvidos no processo geral de vendas demonstrou, portanto, certa melhora após a realização do exercício hands-on. As Figuras 7 e 8 mostram os dois momentos do grupo que exibiu a maior evolução, passando de não identificar nenhum dos atores envolvidos (Figura 7) a, no segundo momento, identificá-los com mais clareza e coerência (Figura 8).

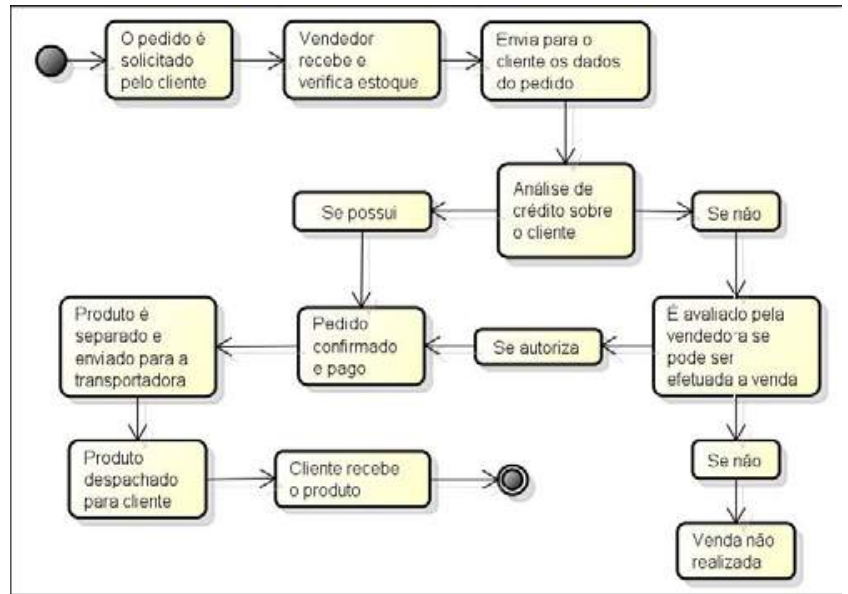

Figura 7 - Diagrama de Atividades modelado pelo Grupo 5 antes da utilização do sistema do sistema ERP.

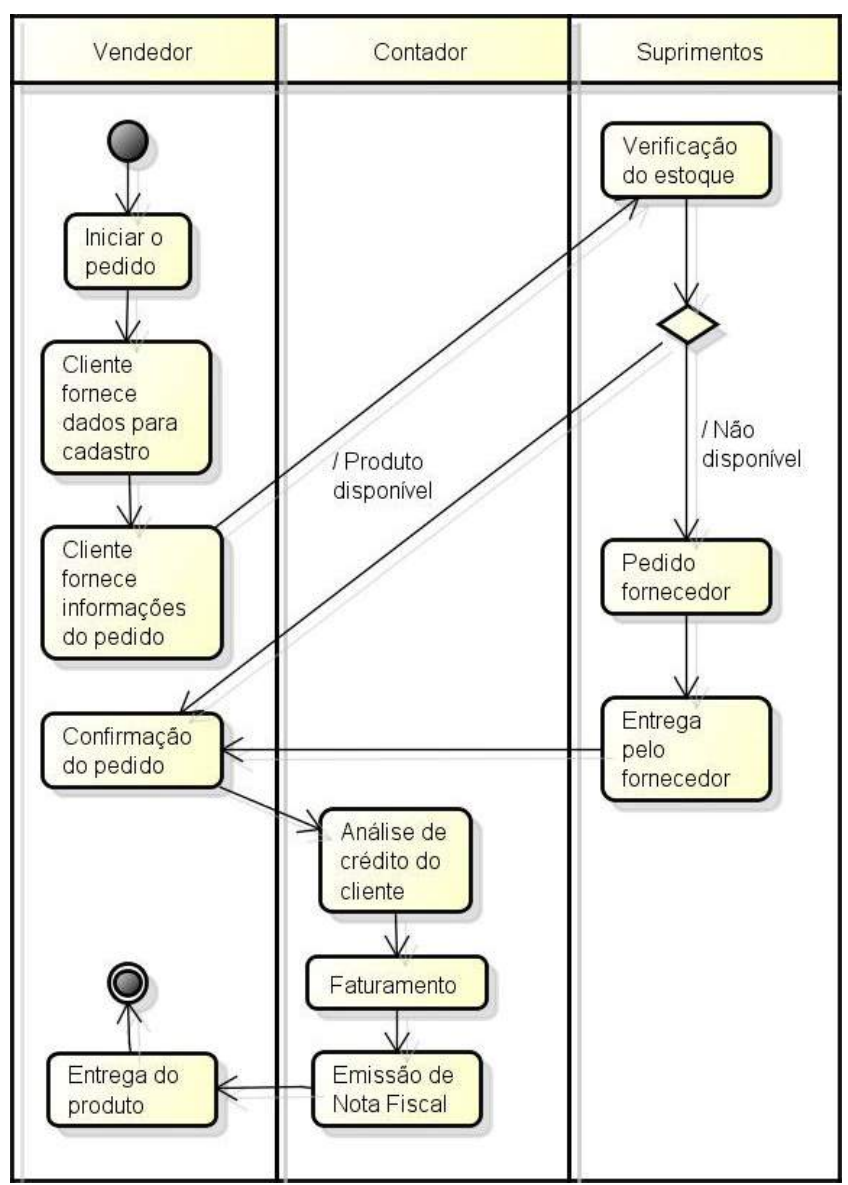

Figura 8 - Diagrama de Atividades produzido pelo Grupo 5 depois da utilização do sistema ERP.

\section{CONSIDERAÇÕES FINAIS}

O presente estudo foi realizado em caráter exploratório, buscando um contato inicial com as possibilidades de utilizar sistemas ERP para auxiliar a compreensão de processos de negócio mais gerais. A metodologia hands-on foi utilizada como ferramenta auxiliar no processo de ensino de sistemas ERP para o curso de graduação em Bacharelado em Administração.

De acordo com os resultados apresentados na seção 4, foi possível observar mais coerência nos atores e nas atividades relacionadas a 
um processo geral de vendas. Apesar de a avaliação ter sido subjetiva, as modelagens sugerem que o uso de sistemas ERP podem favorecer a compreensão dos processos de negócio mais gerais. Esse entendimento se faz importante para um curso na área de negócios, uma vez que constitui para o estudante, um aprendizado importante quanto ao funcionamento de uma empresa. Apesar de a modelagem algumas vezes refletir o contexto e o fluxo exposto pelo sistema utilizado, avalia-se que esta consequência não se torne empecilho, principalmente para processos mais comuns (vendas, compras, estoque, contabilidade, etc.) e que estejam dentro de um contexto das melhores práticas adotadas pelo mercado, uma vez que, de maneira geral, os sistemas ERP implementam as melhores práticas. Esta constatação corrobora o que foi exposto na literatura no caso do experimento relacionado ao conhecimento de processos da área de Recursos Humanos [16]. Ao tratar de processos gerais, a utilização de um sistema ERP favoreceu o conhecimento dos estudantes relativo a esses processos. Quanto à utilização de um sistema ERP open source em detrimento a um sistema comercial, presume-se que o entendimento mais geral do processo pode permitir uma diminuição na curva de aprendizado de um sistema para outro. No entanto, necessita-se de estudo mais aprofundados para se afirmar. Desta forma, considera-se necessária a continuidade do estudo, buscando uma metodologia mais objetiva para aferir com maior precisão e significância estatística o real efeito desta abordagem no conhecimento dos estudantes relativo aos atores e processos das empresas e, principalmente, sobre a visão sistêmica de integração destes processos e atores. Esta necessidade fica evidenciada na medida em que constam na literatura pesquisas de metodologia mais objetiva na qual a exposição do estudante à um exercício hands-on utilizando um ERP real não produziram resultados estatisticamente significativos [13].

Constam também na literatura evidências de que a auto avaliação dos estudantes sobre o conhecimento relativo aos processos de negócio das empresas, bem como a integração dos mesmos, tornase mais positiva após a realização de um experimento utilizando um sistema ERP real e a abordagem hands-on [10, 13]. Partindo deste entendimento, pode-se sugerir a adoção de uma metodologia que inclua também uma auto avaliação do próprio conhecimento por parte dos estudantes.

Por fim, devem-se destacar ainda as dificuldades encontradas no estudo. Alguns dos grupos demonstraram certa dificuldade no uso do modelo utilizado para a elaboração do diagrama de atividades. Isto se torna evidente na medida em que alguns grupos foram excluídos da análise final justamente por não terem montado diagramas utilizando corretamente a notação pedida. Pode ser proveitoso para a continuidade do estudo que seja gasto um tempo maior na elucidação deste modelo aos estudantes.

\section{AGRADECIMENTO}

Os autores agradecem o apoio recebido. Processo no 2015/041181, Fundação de Amparo à Pesquisa do Estado de São Paulo (FAPESP).

\section{REFERÊNCIAS BIBLIOGRÁFICAS}

[1] Ayyagari, R. Hands-on ERP Learning: Using OpenERP, an Alternative to SAP®. Journal of Information Systems Education, 22 (2). 123-133.

[2] Christino, J. M. M., Kaiser, E. L. C. B., Ziviani, F. e Oliveira, J. L. R. O Impacto dos sistemas ERP sobre as variáveis estratégicas organizacionais: Um estudo junto às empresas da
Associação Comercial e Industrial de Ji-Paraná-RO. Revista de Administração e Negócios da Amazônia, 5(3). 63-81.

[3] Davis, C.H., Comeau, J. Enterprise integration in business education: Design and outcomes of a capstone ERP-based undergraduate e-business management course. Journal of Information Systems Education, 15(3). 287-299.

[4] De Bruin, T., Rosemann, M. Towards Understanding Strategic Alignment of Business Process Management. in Proceedings of the 17th Australasian Conference on Information Systems, (Adelaide, Australia, 2006), Queensland University of Technology, 6-8.

[5] Freire, F. M. P., Prado, M. E. B. B. Professores construcionistas: a formação em serviço. in I Congresso de Informática Educativa do Mercosul (Campinas, 1996), Universidade Estadual de Campinas, 229-236.

[6] Joseph, G., George, A. ERP, learning communities and curriculum integration. Journal of Information Systems Education, 13 (1), 51-58.

[7] Klaus, H.; Rosemann, M.; Gable, G.G. What is ERP?. Information Systems Frontiers Journal, 2(2), 141-162.

[8] Léger, P.; Using a Simulation Game Approach to Teach Enterprise Resource Planning Concepts. Journal of Information System Education, 17 (4), 441-447.

[9] Moss, G.R. Working with OpenERP. Packt Publishing, Birmingham, United Kingdom, 2013.

[10] Nelson, R, Millet, I. A Foundation Course in ERP and Business Processes: Rationale, Design, and Education Outcomes. in AMCIS 2001 Proceedings (Pittsburg, EUA, 2001), 991-997.

[11] Object Management Group. OMG Unified Modeling Language. Acesso em 18 de fevereiro de 2015, de OMG Website: http://www.omg.org/spec/UML/2.5/Beta2/PDF

[12] OpenERP S.A. Open source business model, Odoo website. Acesso em 4 de novembro, 2014, de Odoo Website: https://www.odoo.com/blog/odoo-news-5/post/our-opensource-business-model-119

[13] Pridmore, J., Deng, J., Prince, B., Turner, D. Enhancing student learning of ERP and business process knowledge with hands-on ERP exercises. In Proceedings of the Southern Association for Information Systems Conference, (Georgia, EUA, 2014), Southern Association for Information Systems, artigo 31.

[14] Rienzo, T., Han, B. Does ERP Hands-On Experience Help Students Learning Business Process Concepts?. Decision Sciences Journal of Innovative Education, 9(2), 177-207.

[15] Rodrigues, N. N., Estrela, N. V. A. Simple Way: Ensino e Aprendizagem de Engenharia de Software Aplicada através de Ambiente e Projetos Reais. In Anais do VIII Simpósio Brasileiro de Sistemas de Informação, (São Paulo, 2012). 
XI Brazilian Symposium on Information System, Goiânia, GO, May 26-29, 2015.

[16] Wagner, W.P., Najdawi, M.K., Otto, J. An empirical Education, 1, 50-63. investigation into the impact of ERP training on crossfunctional education. Journal of the Academy of Business 